\title{
The Ghosts of Galaxies: Tidal Debris in Clusters
}

\author{
Michael D. Gregg \\ University of California, Davis and Institute of Geophysics and \\ Planetary Physics, Lawrence Livermore National Laboratory, USA \\ Michael J. West \\ University of Hawaii, Hilo, USA
}

\begin{abstract}
Gravitational interactions in rich clusters can strip material from the outer parts of galaxies or even completely disrupt entire systems, giving rise to large scale, low surface brightness ghostly features stretching across intergalactic space. The nearby Coma and Centaurus clusters both have striking examples of galaxy ghosts, in the form of $100 \mathrm{kpc}$-long plumes of intergalactic debris. By searching HST archival images, we have found numerous other examples of galaxy ghosts in rich clusters at low redshift, evidence that galaxy destruction and recycling are ubiquitous, important in cluster formation and evolution, and continue to mold clusters at the present epoch. Many ghosts appear in X-ray bright clusters, perhaps signaling a connection with energetic subcluster mergers.

The fate of such material has important ramifications for cluster evolution. Our new HST WFPC2 $V \& I$ images of a portion of the Centaurus plume reveal that it contains an excess of discrete objects with $-12<M_{V}<-6$, consistent with being globular clusters or smaller dwarf galaxies. This tidally liberated material is being recycled directly into the intracluster population of stars, dwarf galaxies, globular clusters, and gas, which may have been built largely from a multitude of similar events over the life of the cluster.
\end{abstract}

\section{Introduction}

Numerous contributions to this symposium have presented exciting research on intergalactic stellar populations in galaxy clusters. These populations contribute a substantial fraction of the total light of a cluster, $20-40 \%$ in a cluster as typical as Virgo (see review by Arnaboldi 2004, this volume). To understand clusters at even a basic level, then, it is necessary to account for the presence of this intergalactic stuff, not only what and how much is there, but how and when did it get there? And how does it affect the continued evolution of the cluster?

A very pretty simulation of cluster formation has been done by Dubinski (1998) ${ }^{1}$. This view of cluster formation involves not only the gathering together

\footnotetext{
${ }^{1} \mathrm{mpg}$ versions available at www.cita.utoronto.ca/ dubinski/bigcluster.html
} 

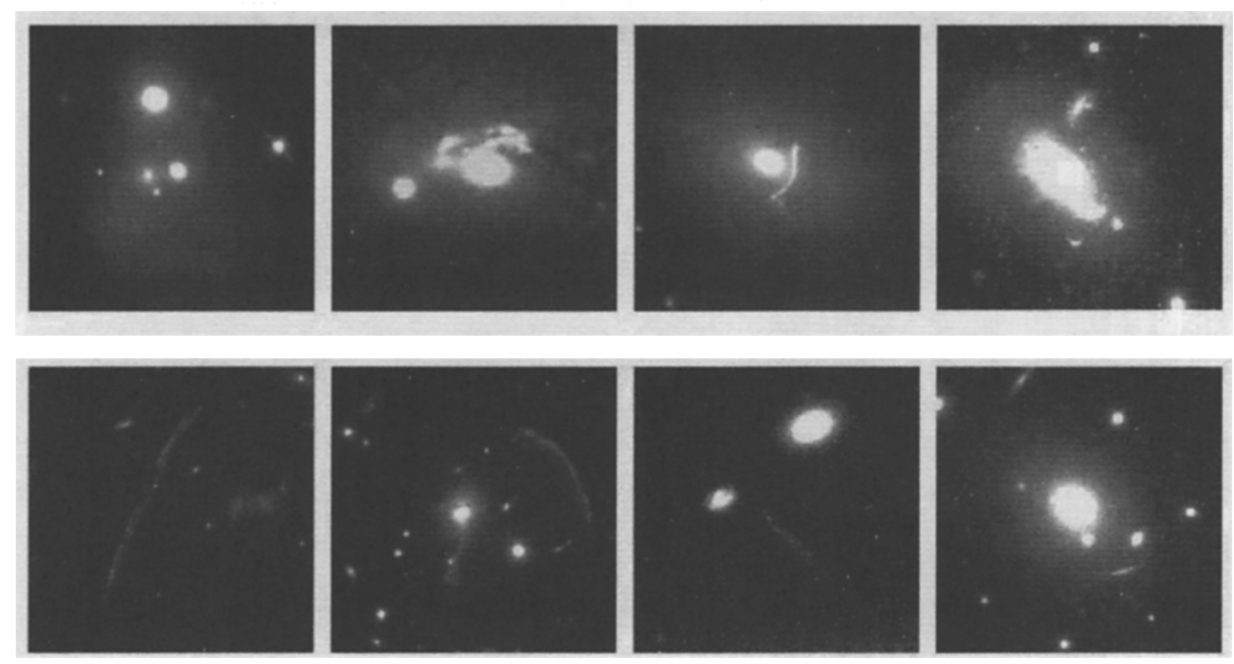

Figure 1. Examples of tidal debris and/or gravitational arcs from archival HST/WFPC2 images of clusters with typical $z \approx 0.1$ to 0.3 . The images are 20-30" large.

of lots of galaxies into close quarters, but the agglomeration of a good many into a single brightest cluster member at the bottom of the global potential. This process requires the disruption, destruction, and recycling of lots of galaxies, giant and dwarf. The intermediate products are distended, low surface brightness splashes of stars across intergalactic space. Fragile, long filamentary plumes and extended pools of stars are quickly extruded and dispersed throughout a cluster, merging into the $\mathrm{cD}$, its extended halo, or the general intergalactic field.

\section{Hunting for Galaxy Ghosts}

Cluster formation continues to the present day, so if the Dubinski simulations are close to the mark, then we should be able to see examples of these ghosts of galaxies: the intermediate, non-equilibrium phases of galaxy disruption and destruction associated with cluster building. One spectacular example is the $100 \mathrm{kpc}$ long, low surface brightness plume in the Coma cluster (Gregg \& West 1998). Only 10-15 kpc wide, it contains roughly the luminosity of the LMC and is probably fated to become part of the halo of NGC4874. While this object seemed unique at first, another plume with nearly identical properties was soon reported by Calcáneo-Roldán et al. (2000) in the nearby Centaurus cluster.

One example of something in astronomy is an anomaly, but two is a population, so we combed through the HST archives looking for more examples of galaxy ghosts. A number of interesting examples turned up, especially among X-ray bright clusters; a few are shown in Figure 1. An immediately obvious complication is that very thin tidal plumes can resemble background galaxies gravitationally lensed by the cluster, and vice versa, especially in ground-based imaging. Some clusters exhibit both phenomena; spectroscopic redshifts are 


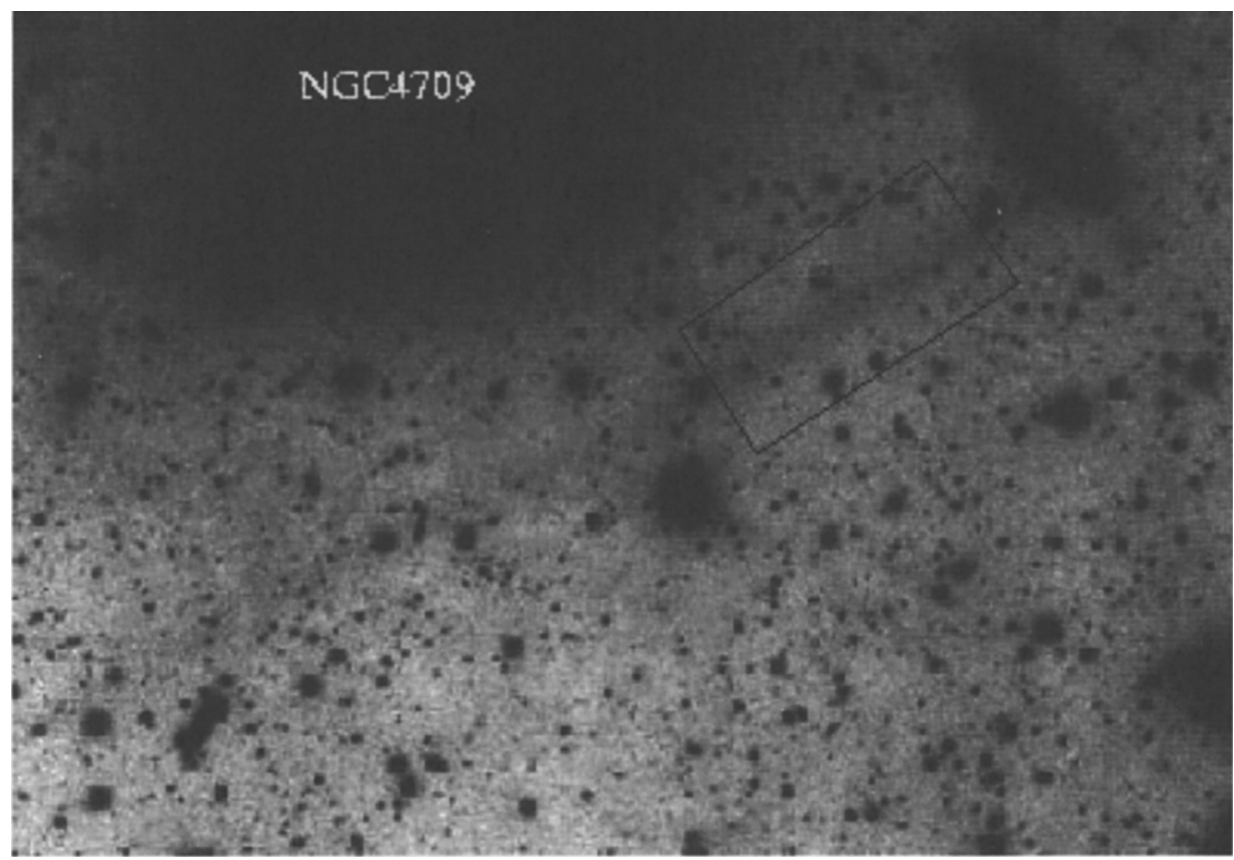

Figure 2. Discovery image of the Centaurus plume (from CalcáneoRoldán et al. 2000). The plume is nearly 8 arcminutes long, over $100 \mathrm{kpc}$ at the distance of Centaurus, extending from the disky galaxy in the upper right down to the lower left edge of the field, probably beyond. The black rectangle shows the location of our WFPC2 images, chips 2 and 3 .

needed for positive identification. Tidally disrupting galaxies can be any color: early types contribute old, red stars and globulars to an intracluster population while gas-rich galaxies may experience a fresh burst of star formation when pulled apart in a cluster. Deep multi-band imaging of these tidal features could provide details of the stellar and star cluster populations which are being strewn through intergalactic space.

\section{WFPC2 Observations of the Centaurus Galaxy Ghost}

Analysis of ROSAT images by Churazov et al. (1999) shows that the NGC 4709 and its entourage are violently colliding with the main body of Centaurus, centered on NGC 4696. The velocity difference of the two components is large, $>1500 \mathrm{~km} / \mathrm{s}$, so perhaps it is no coincidence that one of the more striking examples of galaxy disruption is found in Centaurus. The long, narrow, low surface brightness plume of material stretches more than $100 \mathrm{kpc}$ across intergalactic space, arcing past NGC4709 (Figure 2, top, from Calcáneo-Roldán et al. 2000).

To investigate the Centaurus plume in finer detail, we have obtained WFPC2 $V(\mathrm{~F} 606 \mathrm{~W})$ and $I(\mathrm{~F} 814 \mathrm{~W})$ band images of a portion (Figure 2); the enhanced 


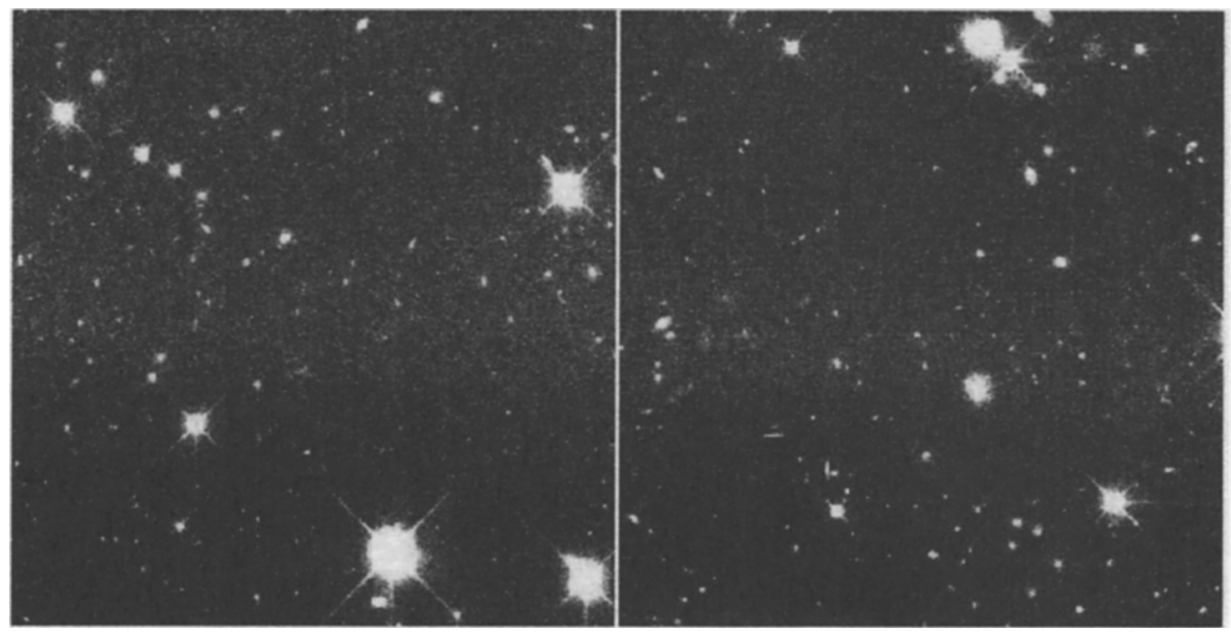

Figure 3. WFPC2 Chips 2 and 3 of our $V+I(F 606 W+F 814 W)$ observations of the Centaurus tidal debris plume. Total integration time is $4800 \mathrm{~s}$ in each filter. The plume is visible as a horizontal stripe of enhanced surface brightness. A mild gradient imposed by the outer halo of NGC4709 (lying off the page to the top) is also present.

surface brightness of the plume is clearly visible in the WFPC2 data (Figure 3). These images reveal that the plume is not a completely smooth, low surface brightness feature, but contains dozens of distinct objects. Star clusters and dwarf galaxies are known to form from tidal debris plumes in field galaxies (Duc \& Mirabel 1994; Duc et al. 2000; Whitmore \& Schweizer 1995; Charlton et al. 2000 ), often accompanied by new star formation. In Centaurus, a similar process appears to be at work generating intracluster populations of stars, globulars, and perhaps even new dwarf galaxies.

\subsection{Identification of Plume Objects}

An object catalog was produced using SEXTRACTOR (Bertin \& Arnouts 1996) on the summed $V+I$ image. The numbers of objects as a function of vertical column (along the plume in Figure 3 ) is statistically consistent with a constant distribution, but the distribution as a function of horizontal row (perpendicular to the plume) is very peaked (Figure 4), differing from constant with high significance, $>99.999 \%$ in a $\chi^{2}$ test. The median filtered rows and columns of the images (Figure 4, lower panel) shows that the diffuse low surface brightness light reflects the peak and shape of the object distribution, confirming the association between the excess numbers and the plume feature. A mild gradient from the outer halo of NGC4709 has been removed from the median-filtered row plot.

\subsection{Luminosity Function and Color Magnitude Diagram}

The Luminosity function of objects in the plume (Figure 4) compared to adjacent fields (scaled to an equivalent area) shows that the excess is mainly in the luminosity range $M_{V} \approx-7$ to -9 , but there are extra objects as bright as 

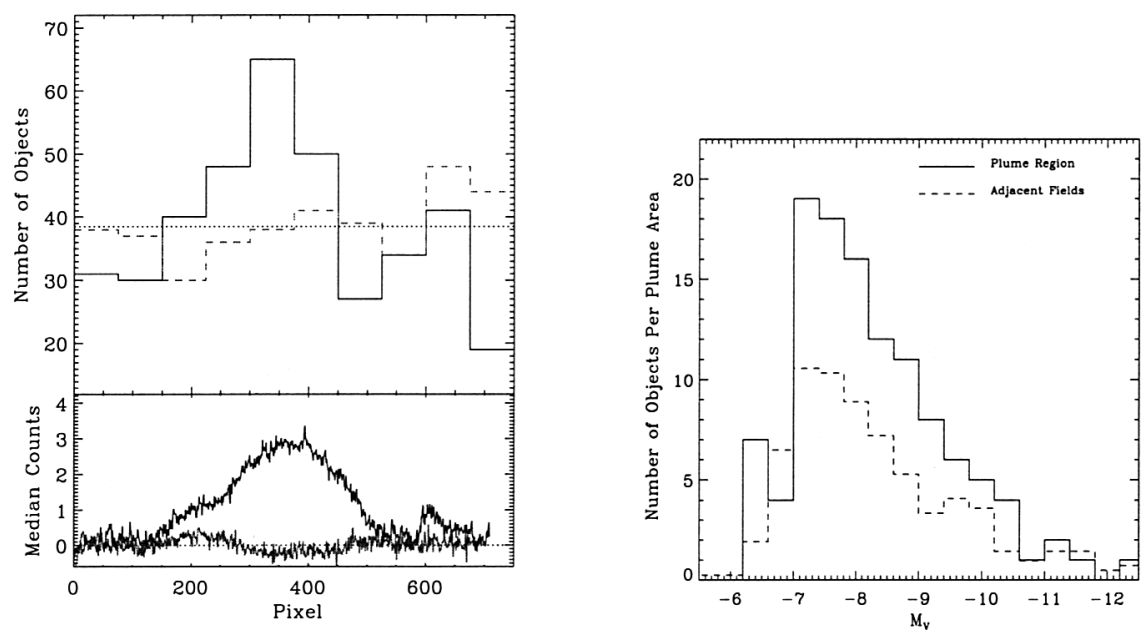

Figure 4. Left: distribution of discrete objects in the WFPC2 plume images as a function of row (solid histogram) and column number (chips 2 and 3 done separately). The distribution peaks strongly in the plume, but is roughly constant in the orthogonal direction. The lower plot shows the median of vertical columns (black) and horizontal rows (grey); the peak in object numbers occurs in the highest surface brightness portion of the plume. Right: Luminosity function of objects in and out of the plume; these are in the luminosity range of globular clusters and small dwarf galaxies.
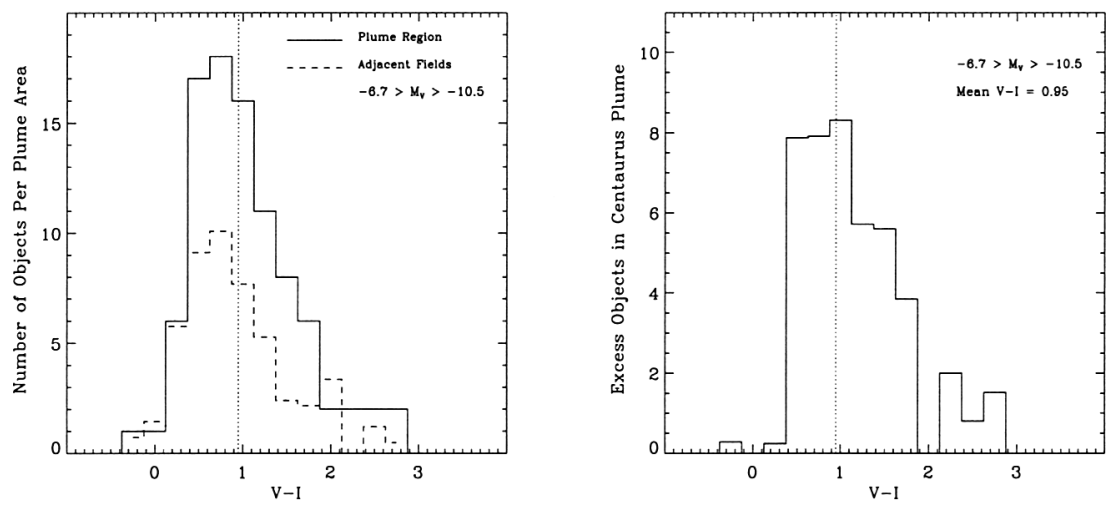

Figure 5. Left: Color-magnitude diagram of plume and non-plume objects. Right: Difference histogram showing that the plume objects have an average color of $V-I=0.95$, typical of globular clusters in large ellipticals. 
$M_{V} \approx-12$. (We adopt $M-m=33.4,47 \mathrm{Mpc}$, for Centaurus.) These luminosities are in the range of globular clusters and faint dwarf galaxies; the excess consists of both extended objects and point sources. If these objects are bound and can survive the disruption event, then we are witnessing ongoing creation of dwarf galaxies from the shards of a larger object and the injection of old globular star clusters into intergalactic space.

There are 91 objects in the plume, and only $\sim 50$ in the area-scaled field. A 'Median-Delta' test shows that the difference of the two is not random at a significance level of 0.0004 . These numbers predict that the entire plume (extending far to the east of our WFPC2 field) contains $\sim 80-100$ objects.

A comparison of the color-magnitude relations for objects in and out of the plume (Figure 5) shows no discernable difference in color distributions. Ordinary globular clusters in these bands have $V-I \approx 0.95$ (e.g. Kavelaars et al. 2000), consistent with the distribution of plume (and non-plume) object colors. The plume is in the outer halo of NGC4709; perhaps many of the non-plume objects are also globular clusters and faint Centaurus dwarfs. Most of the objects are consistent with being point sources, so some could be individual supergiants, which would imply recent star formation.

\section{Discussion and Summary}

- In the Centaurus plume, we are witnessing the present-epoch creation of intergalactic objects in a rich cluster. The stripped material, now floating in the general cluster potential, will eventually disperse throughout the core of Centaurus, augmenting the halos of giant ellipticals and the intergalactic populations of stars, star clusters, and dwarf galaxies.

- Any interstellar gas and dust associated with these objects is added to the hot intracluster medium, already bright in the X-ray.

- The tidal generation of new dwarf galaxies can explain the observed association of excess faint galaxies with dynamically turbulent clusters (e.g. Lopez-Cruz et al. 1997).

- Disruption of infalling galaxies and the liberation of tidal debris is an important driver of the evolution of member galaxies the cluster as a whole.

- Objects such as the tidal plume in Centaurus provide a glimpse of a more chaotic era when today's rich galaxy clusters were beginning to form.

Acknowledgments. This work is based on observations made with the Hubble Space Telescope; support was provided by NASA through grant GO8644 from the Space Telescope Science Institute, operated by AURA, Inc., under NASA contract NAS5-26555. Part of this work was performed under the auspices of the U.S. Department of Energy by University of California Lawrence Livermore National Laboratory under contract No. W-7405-Eng-48.

\section{References}

Bertin, E. \& Arnouts, S. 1996, A\&AS, 117, 393 
Calcáneo-Roldán, C., Moore, B., Bland-Hawthorn, J., Malin, D., \& Sadler, E.M. 2000, MNRAS, 314, 324

Charlton, J., Knierman, K., Hunsberger, S., Gallagher, S., Whitmore, B., Kundu, A., \& Hibbard, J., astro-ph/0009196

Churazov, E., Gilfanov, M., Forman, W., \& Jones, C. 1999, ApJ, 520, 105

Dubinski, J. 1998, ApJ, 502, 141

Duc, P.-A., Mirabel, I.F. 1994, A\&A, 289, 83

Duc, P.-A., Brinks, E., Springel, V., Pichardo, B., Weilbacher, P., \& Mirabel, I.F. 2000, AJ, 120, 1238

Gregg, M.D. \& West, M.J. 1998, Nature, 396, 549

Kavelaars, J. J. 2000, ApJ, 533, 125

Lopez-Cruz, O., Yee, H.K.C., Brown, J.P., Jones, C., \& Forman, W. 1997 ApJL 475, L97

Whitmore, B.C., Schweizer, F. 1995, AJ, 109, 960 